\title{
EDITORIAL
}

\section{A "Seasonal Stew" - Food for Thought; Fuel for Action}

\section{William H. Barton}

Welcome to the Fall 2013 issue of Advances in Social Work. Arriving on the heels of our previous, 17-article special issue on Latinos/Latinas, we now present the largest regular issue (21 articles) ever published by Advances. For this, we must thank the many authors who submitted manuscripts during the last several months, as well as a tireless cadre of reviewers who typically view and evaluate each eventually successful manuscript at least two times prior to its acceptance. We appreciate the increase in submissions and the growth in our ranks of registered authors, readers, and reviewers. We now have more than 800 social work researchers, educators, and practitioners from around the world registered with the journal, about 300 of whom serve as reviewers.

The sheer number of articles in this Fall 2013 issue precludes the usual practice of individually introducing each one here in detail. Instead, what follows are descriptions of several loose groupings of these articles. The first four articles should be of broad interest. The first, by Chaumba, presents a classification and list of mixed methods studies in social work, organized by the reasons mixed methods were chosen. The second, by Strand, Hansen, and Courtney, extracts common elements across several evidence-based trauma treatment protocols. The third, by Dybicz, contrasts traditional, problem-oriented and postmodern, strength-based practice approaches in terms of their respective approaches to gathering information from clients. The fourth, by Powell, Garrow, Woodford, and Perron, suggests a variety of ways direct practice social workers can influence policy.

Next is a set of articles concerned with specific populations. Hodge and Limb discuss using spiritual histories in practice with Mormons, or Latter-day Saints. Shier and Graham focus on how community-based organizations can identify and respond to social service needs of Muslims in the post-9/11 era in the United States. Fasbinder, Monson, Montero, Sanders, and Williams derive implications for social work with same-gender partners from an analysis of published survey data from 1996 to 2013 tracking public attitudes towards same-gender marriage and civil unions. The next article, by Wall, takes a more direct approach to offer suggestions for social worker practitioners and agencies working with lesbian-headed families. The last article in this group, by S. Young and McLeod, addresses strategies for HIV/AIDS prevention and intervention with Black men who have sex with men.

The following two articles contain the results of survey research studies. Haffejee, Yoder, and Bender report on results from a mixed methods study of a sample of college students aged 18-25 examining factors differentiating those who abstained from illegal behaviors, desisted from illegal behaviors, and persisted in illegal behaviors. Castillo, Asante, Dwumah, Barnie, and Becerra present results from their survey of Ghanaian social work students' perceptions of poverty and social welfare policies in Ghana.

William H. Barton, Ph.D., is a professor in the Indiana University School of Social Work on the Indiana University-Purdue University Indianapolis campus. Contact information: (317) 274-6711; Email: wbarton@iupui.edu

Copyright (C) 2012 Advances in Social Work Vol. 14 No.2 (Fall 2013), i-ii 
Three diverse conceptual articles comprise the next group. Sutphin, McDonough, and Schrenkel argue that formal theories are relatively rarely used to guide social work research. By using Family Systems Theory as an example, they suggest that formalization of theories can usefully guide social work research and practice. Next, J. Young presents a conceptual model to describe how contemporary nonprofit organizations can use social media as part of their organizational identity construction. Organizations are also the focus of Majiros' article that examines principles of social exchange theory associated with mentoring as a means of knowledge transfer in the federal workplace.

The final group of seven articles is related to social work education. McCave, Rishel, and Morris present the second in a two-part series of articles (the first appeared in Advances in the Fall 2011 issue) decrying the relative absence of prevention-focused language and content in social work curricula, textbooks, and presentations at national social work conferences. Park, Hawkins, Hawkins, and Hamlin examine attitudes towards interprofessional collaboration among a sample of medical, nursing, and social work students, with implications for expanding interprofessional education efforts. The next two articles focus on styles of leadership. Goldkind and Pardasani surveyed a sample of human services executive directors to explore the relationship between academic background (i.e., social work vs. other disciplines) and leadership style. Call, Owens, and Vincent present a qualitative analysis from a survey of social work faculty members about how they experienced the leadership style of their current academic unit heads. Hunter and Mileski offer a case study of academic civic engagement, the formation and impact of the Emerging Leaders Project, a community-based participatory action research (CBPR) project focused on capacity building with new arriving communities in Salt Lake City. Turning to the classroom, Acquavita and Tice discuss lessons learned from incorporating a process of reflection and peer review in a social policy course. Closing out our Fall issue is a critique of neo-liberal social work education from our colleagues to the North (Canada) - George, Silver, and Preston - who argue for nothing less than a reconceptualization of social work field education that could result in a more transformative educational experience.

There you have it, a seasonal stew - food for thought and, perhaps, fuel for action in the realms of social work practice, research, and education. You may have noticed from the announcement on the home page of Advances in Social Work that our Spring 2014 issue will be very special, indeed. "Eyewitnesses to History: First-Hand Accounts of Sages of the Profession," under the guidance of co-editors Drs. Virginia Majewski and Gerald T. Powers, will consist of a series of invited articles concerning "sages of the profession," individuals who are generally recognized as intellectual and professional pioneers in the field of social work. Then, as usual, we will produce a "regular" issue with a range of topics in the Fall of 2014. Look for a call for papers this spring or summer for a 2015 special issue on "Technology, the Internet, and Social Work Practice."

I hope you enjoy the current issue, work on those manuscripts to submit, tell your colleagues about Advances in Social Work, and urge them to register to submit articles and to join our growing corps of reviewers.

\section{Happy Holidays!}

